\title{
Where does intraoperative neurophysiological monitoring stand in canada in 2016 ?
}

Volume 5 Issue 4 - 2016

\author{
Keywords: IONM, Canada, CANM, technologists, \\ neurophysiologists, interpretation, practice guidelines, service \\ delivery model, liability, education and training
}

\begin{abstract}
Abbreviations: AMA, american medical association; ASNM, american society of neurophysiological monitoring; CANM, canadian association of neurophysiological monitoring; IONM, intraoperative neurophysiological monitoring; MD, medical doctor; MSc, masters of science; $\mathrm{PhD}$, philosophy doctor; USA, united states of america; OR, operating room
\end{abstract}

\section{Introduction}

A recent article published in the Canadian Journal of Surgery by Norton JA et al., ${ }^{1}$ has drawn the attention of the IONM community, especially in Canada. In October 2015, the American Society of Neurophysiological Monitoring (ASNM) eNewsletter The Monitor posted a letter by the Canadian Association of Neurophysiological Monitoring (CANM) arguing against Norton et al.'s published findings. ${ }^{2}$ As a fellowship-trained and board-certified attending clinical neurophysiologist practicing in one of the major neurological surgery centers in Canada, this letter is intended to express my own point of view regarding the current state of IONM in Canada in an effort to open the channels of communication and help resolve this schism.

\section{Norton JA et al.,'}

Briefly, Dr. Norton and his colleagues sent a survey to Canadian spine-, neuro-, cardiovascular, and ENT surgeons asking who currently interprets their IONM data, and who would they prefer to interpret the data were they given the choice. ${ }^{1}$ The clear finding from the responses of 227 surgeons was that the majority of Canadian surgeons across all specialties were interpreting their own IONM data (> 63\%), but most would prefer not to. When asked who they would prefer to interpret IONM data, surgeons responded with a strong preference for $\mathrm{PhD}$ Neurophysiologists $(92.7 \%)$ and MD Neurologists (74.3\%), followed by self $(9 \%)$ and Technologists/MSc-Technologists $(0 \%)$. The rebuttal letter published by CANM in the ASNM eNewsletter claims the questions asked in Norton's survey were biased $;{ }^{3}$ however, CANM provided no evidence in support of this claim. In contrast and after verifications, Norton confirms that all of the survey questions were reviewed by a range of professionals in Edmonton, including medical experts from the Canadian Royal College of Medicine.

\section{Technologist vs. Attending Clinical Neurophysiologist}

It may not be surprising that CANM, with a majority of members being practicing technologists, would oppose the results of Norton's survey which shows that Canadian surgeons do not want technologists to interpret IONM data. For many years, CANM has promoted a "technologist-only" service delivery model for IONM in Canada:

"In contrast to the oversight system proposed in the United States, CANM believes best patient care occurs when there is a highly qualified and trained person performing IONM in the OR who interprets and

\author{
Sebastien Fournier \\ Department of Surgery, Université Laval, Québec, Canada
}

Correspondence: Dr. Sebastien Fournier, CHU de Quebec Université Laval, I4I, I8e Rue, office J-252 (OR), Québec City, Québec, Canada,Tel 418-649-0252; ext.: 63740,

Email sebastien.foutnier.cha@ssss.gouv.qc.ca

Received: November 16, 2016 | Published: December 13, 2016

communicates IONM findings to the surgeons in a timely manner, and who participates in recommendations for intervention allowing for optimal patient surgical safety" 1

By this statement CANM suggests that the oversight IONM system widely used in the United States does not provide the best patient care. In contrast, CANM claims that IONM practitioners in the OR (mainly technologists) are "qualified to interpret" IONM data which raises serious concerns about the level of education, training, experience and overall capabilities of any person expected to practice IONM at the level of IONM data interpretation and to provide the surgeon with a differential diagnosis and recommended interventions, in other words, to practice medicine. The true basis and fundamentals of best patient care lie in education and thorough training. A "highly qualified and trained person" is a non-specific term unless it is supported by higher level education and fellowship/residency training. Perhaps the only relevant definition here is that of competency and in this sense CANM needs to further define what is meant by "optimal patient surgical safety".

Furthermore, CANM vigorously argues against either the remote or in-person professional oversight models that are prevalent not only in the United States, but in most countries. Again, this argument is not supported by evidence and is solely based on the false premise that these traditional models cause a delay in responsiveness and that an immediate response by a technologist devoid of any data interpretation amounts to adequate neurophysiological monitoring:

"With the backing of its membership and other healthcare providers, CANM is moving forward with a system that will recognize 
IONM professionals in Canada as self-regulating independent practitioners. By elevating the standards for practice of IONM, patients will be afforded with a system that negates the need for "interpreter" oversight (be that from a PhD, neurologist, or any other professional) and will be provided with true real-time interpretation of neurophysiological signals by the IONM professional present in the operating room... There has been much debate over whether the interpretation of IONM signals constitutes the "practice of medicine" although no matter which side of the argument one falls, there can be no debate over the fact that neurophysiological signal abnormalities require prompt recognition and immediate interventional actions in order to avoid harm to the patient. ${ }^{4}$,"

All IONM practitioners, regardless of their role, will agree that the immediate detection of significant deviations in IONM data is of prime importance. However, CANM has never established that a prompt and accurate interpretation of data by an attending clinical neurophysiologist, either in-house or remotely, is not the current practice in Canada (or elsewhere), nor have they established that a less qualified technologist is capable of rendering an accurate and complete diagnosis. Until proven otherwise, the technologist-only model is expected to increase the number of inaccurate and falsepositive alarms, which over time would result in a loss of credibility of the IONM service. Ultimately, this will lead to surgeons choosing not to utilize IONM services in future cases based on the premise that "bad monitoring is worse than no monitoring". Indeed, Norton's survey showed that self-monitoring is preferred by $9 \%$ of surgeons and that none $(0 \%)$ prefer the technologist-only model. Although CANM may have an inclination to support and defend the interests of its technologist members, the highest priority for Canadian patients is to provide the service delivery model for IONM that is based on published evidence and not opinions or assumptions. To date, many publications that have shown the value of IONM at improving patient outcomes have been based on the traditional service delivery model that includes a certified technologist working under the direct supervision of an attending neurophysiologist. 5

CANM may try to take advantage of the fact that in comparison to the USA, IONM is not regulated by the Canadian federal and provincial governments. As a consequence, across the country, the practice of IONM complies with no approved standards or guidelines. CANM, which has self-proclaimed to be the national body representing IONM practitioners in Canada. ${ }^{2}$ has never advocated any standards, guidelines, policies and procedures to its members and yet, has now proposed the technologist-only model as the "best patient care" servicedelivery model without even receiving overall support amongst Canadian technologists. In fact, several very experienced technologists have voiced feeling uncomfortable about interpreting IONM data and communicating findings. The reason for this is that surgeons typically want to hear more than a simple alert (e.g.: $50 \%$ decrease in amplitude for SSEPs). They expect to engage in a peer-to-peer discussion of the potential causes that matched to the electrophysiological data and to the preceding surgical act, and to have the IONM professional propose a plan of intervention. As I understand it, this would be an obvious source of stress to technologists who lack the qualifications and comprehension of surgical procedures, anatomy, neurophysiology and hemodynamics, all of which in the practice of medicine are components essential to maintain effective communication with the surgeon.

\section{Medicolegal responsibility and accountability}

It is also required to define who assumes medical liability in any service delivery model. CANM's current President - Elect dodged this question by asserting that:
"In the challenging environment of the operating room, the goal of the neuromonitorist is not diagnosis, but the evaluation of deviations from a set of baseline neurophysiological tests. It is the neuromonitorist's task to identify any deviations from the baseline data set and establish a timely liaison between relevant clinical professionals, which predominantly includes the surgeon and/or anesthesiologist. In this respect, actions taken to rectify the changes in neuromonitoring data are placed in the hands of the surgeon or anesthesiologist". ${ }^{3}$

There are two significant problems with this statement. First, this contradicts CANM's earlier statement which stipulates that:

"CANM believes best patient care occurs when there is a highly qualified and trained person performing IONM in the $O R$ who interprets and communicates IONM findings to the surgeons in a timely manner, and who participates in recommendations for intervention allowing for optimal patient surgical safety". ${ }^{2}$

The interpretation of IONM data (whether in person or remotely) is the act of arriving at a differential diagnosis. CANM's stated positions are inconsistent and do not define who is qualified to provide a medical diagnosis and as a consequence, who is liable for the medical outcome that is based on this diagnosis. Second, in the setting of the technologist-only model, the surgeon alone will assume the liability for the patient outcome, whether it is the technologist and/or the surgeon who is interpreting the IONM data and making a medical diagnosis. The service delivery model in the USA confers liability for the interpretation of IONM data on the IONM team and not on the surgeon and with the exception of ENT surgeons, most surgeons are not privileged in their scope of practice to interpret IONM data. The CANM proposal assumes that Canadian surgeons will accept all medical liability while the technologist assumes none. Although this status quo approach seems to position CANM in a no-risk no-benefit position as regards patient care, Canadian surgeons have clearly expressed their wishes to benefit from the services of qualified and privileged Ph.D. or M.D. clinical neurophysiologists.

According to the American Medical Association, Policy H-410.957, IONM is the practice of medicine and its interpretation requires a suitably qualified practitioner (Policy H-35.971 and confirmed by the IONM Workgroup of the AMA in 2013). This policy was referenced by Norton et al. ' in the author response to Wilkinson \& Kaufmann's letter to the editor. ${ }^{3}$ However, because there are no standards of care for IONM in Canada and IONM has not been declared to be the practice of medicine, the vast majority of surgical centers in Canada do not use the services of a qualified clinical neurophysiologist. It is a paradox that given the advanced nature of IONM in the USA compared to IONM in Canada, it is incomprehensible that CANM, without a shred of evidence to support its position, is opposed to applying the established and regulated American service delivery models to Canada. In response to Wilkinson \& Haufmann, ${ }^{3}$ Norton et al., ${ }^{1}$ wrote:

"The IOM situation should not be different from that in the outpatient laboratory. A skilled individual performs the technical aspect of the task and provides an impression of the test. The test itself is interpreted by a clinical practitioner. The patient's physician (most responsible physician; MRP) integrates this interpretation with other clinical signs and knowledge to form a diagnosis and treatment plan. Indeed, many reports from the electroencephalography laboratory (or the radiology department, which also follows this model) end with the statement that "clinical correlation is required." In the intraoperative neuromonitoring (IOM) setting we see this as being a Certification of Neurointraoperative Monitoring (or a Canadian equivalent when/ if available) technologist placing electrodes, running the tests and 
providing an impression and a doctor or clinically trained doctorallevel neurophysiologist providing real-time interpretation to the surgeon (MRP). It is the surgeon's responsibility to decide what to do with the interpretation". ${ }^{3}$

It is worthy to note that Canadian patients are becoming more and more informed about surgical outcomes and medical malpractice For better or for worse, patients are becoming more litigious, as represented by an increased number of lawsuits across Canada, judging surgeons and in extension IONM technologists, accountable for medical malpractice. Often, this is the result of a surgeon and a technologist having limited knowledge of and/or training in IONM.

\section{Educational program vs.Training}

In recent years, CANM has created a partnership with the Michener Institute in Toronto to establish a 2-year online IONM Graduate Certificate Program ${ }^{2}$ :

"CANM in concert with the Michener Institute for Applied Health Sciences initiated an education and internship program leading to a national examination and certification (see www.canm.ca) to ensure quality of the IONM practitioner. This program is open to those with a minimum bachelor's degree in a health science related discipline (including MDs and PhDs)".

However, according to the program requirements, the course is open to the following individuals, some of whom do not possess a bachelor's degree and have very limited knowledge of IONM:

1. Currently practicing intraoperative neurophysiological monitoring with an accredited hospital or;

2. Full CANM membership (please visit the CANM website for membership criteria) or

3. Bachelor's degree in a health-related field (with preference given to courses in human anatomy or other health sciences).

The prerequisite listed as number 1 could mean that anyone irrespective of academic background with barely any exposure to IONM (which could be interpreted as a minimum of 1 IONM case), is eligible to register for this program. This, by definition, is not a graduate program. Moreover, this educational program is open to students worldwide. According to this year's listing of students, the majority are overseas students and thus unlikely upon graduation to meet Canada's IONM needs. And foremost, CANM falsely claims to have initiated an internship program in IONM. There are currently no training centers in Canada with pathways leading to a national practical examination, certification and recognition. As it stands, graduates of the Michener program are only exposed to 6 online theoretical courses without any exposure to the practical reality of the operating room. Despite efforts to establish a university program in IONM, there are still no real accredited IONM training programs in Canada.

\section{Responding to the limited availability of IONM practitioners}

In a recent letter entitled The Economics of IONM Expertise: When Supply Falls Short of Demand, Dr. Richard Vogel ${ }^{6}$ writes about the imbalance between the supply of qualified neurophysiologists in the setting of increasing demand for IONM services in the USA. Canada faces perhaps an even more severe imbalance related to an ongoing increase in demand for IONM services and the need for both highly trained technologists and Clinical Neurophysiologists, whose number is currently around a dozen across the country.

According to my humble opinion, the severe shortage of clinical neurophysiologists in Canada is not an excuse to promote the technologist-only model and expose patients to sub-standard IONM services. Rather, the traditional IONM service delivery model which involves a qualified attending-level clinical neurophysiologist working with a certified technologist should be adopted in Canada. This has proven to be successful because it is based on extensive education, training and regulation through appropriate credentialing. ${ }^{5}$ To increase the supply of qualified clinical neurophysiologists, as it is in the USA, Canada should broaden the scope of practice to permit non-physician and board-certified health care professionals such as Chiropractors and Audiologists to provide professional IONM services. Or, as Dr. Vogel pointed out in his letter, there are several highly trained neuroscientists in Canada struggling to secure a spot in academia. They have the educational background and critical thinking skills to be great IONM practitioners and could potentially be attracted by the clinical work of IONM. It is our responsibility to reach out and inform them about clinical neurophysiology. Alberta, for example, has had 7 doctorate level scientists join IONM and receive thorough training; 3 of them are still currently active in Alberta as full-time clinical neurophysiologists according to chief neurophysiologist, Dr. François Roy. Dr. Roy confirms finding it easy to recruit and train $\mathrm{PhD}$ students as they're all eager to participate in IONM. Therefore, this type of recruitment and training assures a high level of knowledge and competency in the OR. Again, currently in Canada, the training of new IONM practitioners is left to each individual center; some of them have gone extra steps to guarantee high level patient care.

\section{Conclusion}

In conclusion, I believe that all Canadian IONM practitioners (technologists and neurophysiologists) should strive to provide the best possible patient care. This letter was intended not to further divide but to initiate a national dialogue to define the appropriate service delivery model for IONM in Canada; one that includes all stakeholders and explores how to achieve the best patient care, and one that specifies the members and credentials of the IONM team. As a growing Canadian IONM community, we should respect the wishes of surgeons and work together to provide them with what many other countries, especially the USA, has deemed to be the best service delivery model for the practice of IONM.

\section{Acknowledgments}

None.

\section{Conflicts of interest}

None.

\section{Funding}

None.

\section{References}

1. Norton JA, Aronyk KE, Hedden DM. Interpretation of surgical neuromonitoring data in Canada: a survey of practising surgeons. Can $J$ Surg. 2015;58(3):206-208.

2. ASNM. Canadian Association of Neurophysiological Monitoring, Toronto, Canada; In ASNM Monitor eNewletter, October 2015. 2015

3. Wilkinson M, Haufmann K. Interpretation of surgical neuromonitoring data in Canada: our view. Can J Surg. 2015;58(6):E4.

4. CANM. Canadian IONM News. 3(1): Andrew John publishing, Canada 2014.

5. Skinner SA, Cohen BA, Morledge DE, et al. Practice guidelines for the supervising professional: intraoperative neurophysiological monitoring. J Clin Monit Comput. 2014;28(2):103-111.

6. Vogel R. The Economics of IONM Expertise: When Supply Falls Short of Demand; In ASNM Monitor eNewletter, June 2015. 2015. 